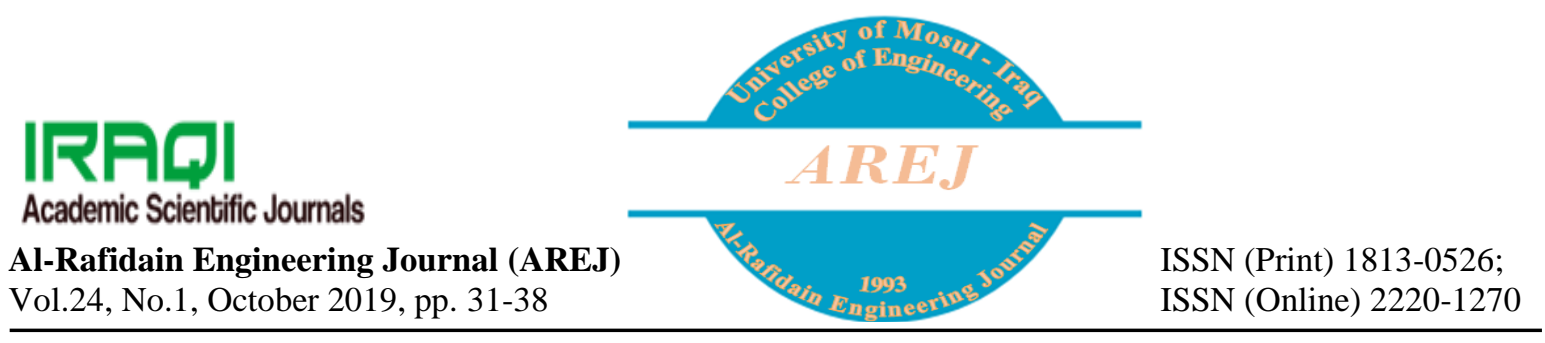

تأثير تخشين مطغى الفيضان في القناة المركبة المتناظرة على مقاومة الجريان

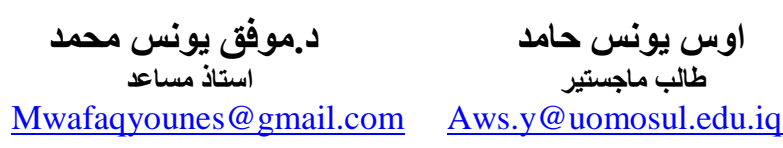

قسم هندسة السدود و الموارد المائيةـ كلية الهندسة- جامعة الموصل

قبل: 24-1-2019

استلم: 19 -11-2018

بتناول هذا البحث دراسة مختبرية لدراسة تأثبر تخشبن مطغى الفيضان في قناة مركبة متناظرة (القناة الرئبيسة 18 سم ومطغى الفيضان

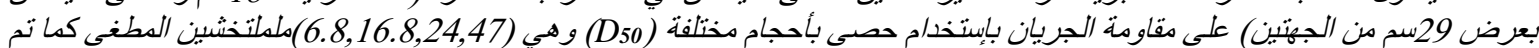
دراسة مقاةمة الجريان على المطغى الغير مخشن. اخنت خمسة تصاريف لكل حالة وتراوح التصريف بين (34,27.8,19.35,12,7.87)

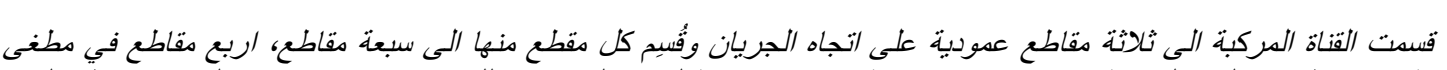

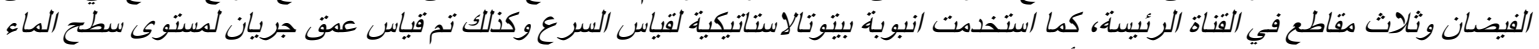

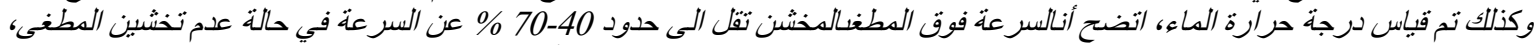

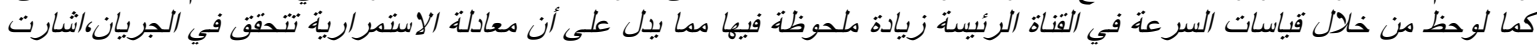

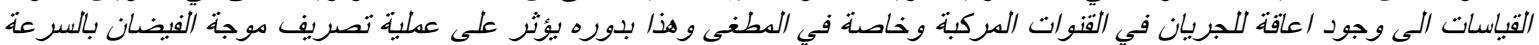

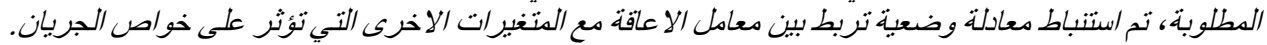

الكلمات اللالهه :

القناة المركبة، الخشونة، توزيع السرعة، التصريف، رقم فرود

https://rengj.mosuljournals.com

Email: alrafidain_engjournal@umosul.edu.iq

الأحيان تكون خشونة القناة الرئيسة أقل من خشونة

1. المقدمة

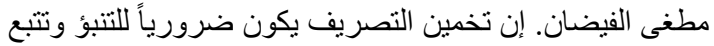

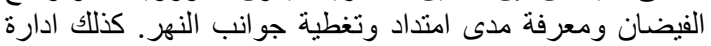

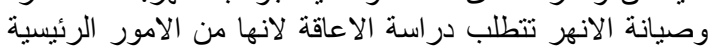

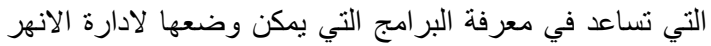

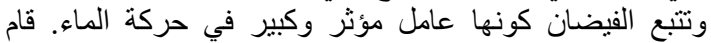

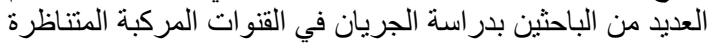

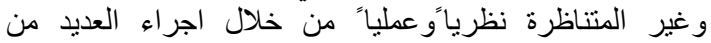

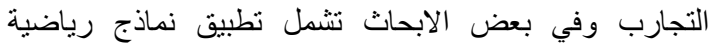

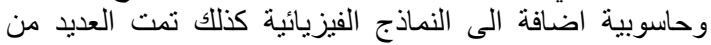

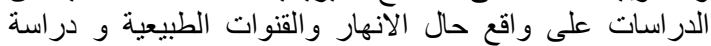

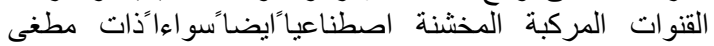

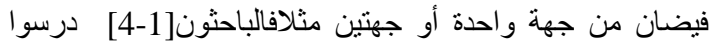

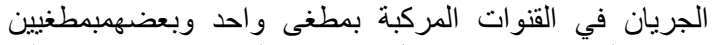

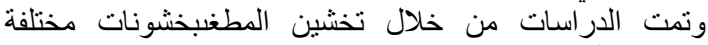

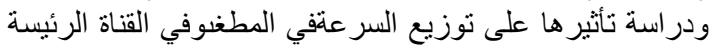

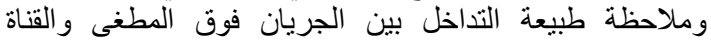

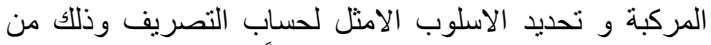

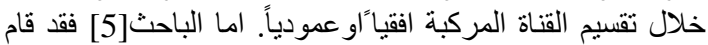

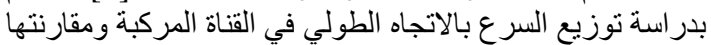
باستخدام اربع موديلات رياضية مختلفة ولاحظ البان الباحث أن هناك ولك

تعرف القناة المركبة بأنها القناة التي تتكون من مقطع

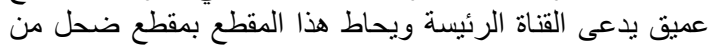

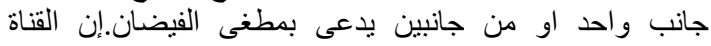

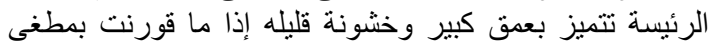

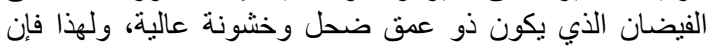

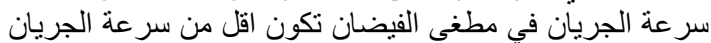

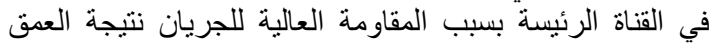

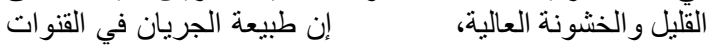

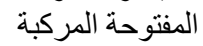

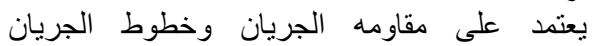

و المتغيرات الهندسية والتداخل بين المطغى والقناة الرئيسة مما لجرئ

الجريان معقد اذا ما قورن مع الجريان في القنوات

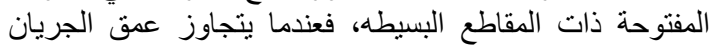

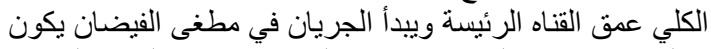

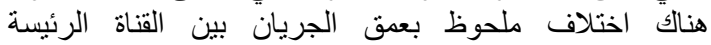

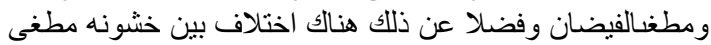
الفيضان وخشونة القناة الرئيسة، في معظم ذلفئ هن 
تم تقسيم القناة الى ثلاث مقاطع عرضية وقسمت هذه فئ الحسابات

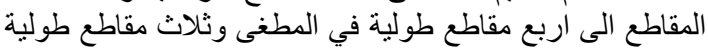
في القناة الرئيسة كما موضح مقاطع بالثكل (2).

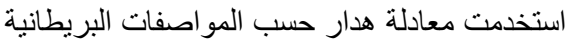
القياسية ( British Standard No.3680:part [4A:1965) وضع مؤخر القناة لحساب التصريف

$$
\begin{aligned}
Q_{b}=2.3594(0.0602 \\
+0.3092 H)(H \\
+0.001)^{1.5} \ldots
\end{aligned}
$$

m³/sec التصريف = Qb

(m) ارتفاع الماء المقاس فوق حافة الهدار

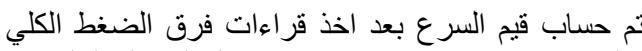

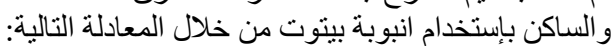

$$
V=\sqrt{2 g \Delta H}
$$

\section{حيث:}

: $\mathrm{m} / \mathrm{sec}$

هH

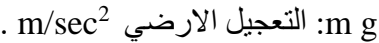

وبإعتبار أن قيمة معدل السر عة على بعد 0.6 من سطح

$V^{\prime}=V_{0.6}$

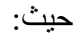

: قيمة معدل السر عة

V 0.6 وبعد ذللك تم حساب قيمة الخشونة من معادلة ماننغ :

$n=\frac{1}{V \prime} R^{2 / 3} S^{0.5}$

n معامل ماننك للخشونة : معدة :

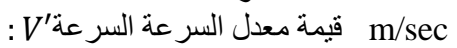

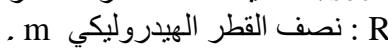

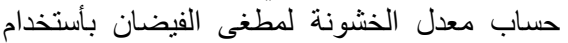

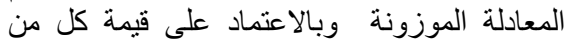
المحيط المبتل و الخشونة في كل مقطع.

$$
n^{\prime}=\frac{n 1 * P 1+n 2 * P 2+\cdots+n_{n} * p_{n}}{P 1+P 2+\cdots+p_{n}} \ldots \cdots
$$

nn

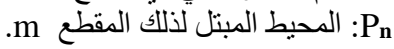

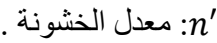

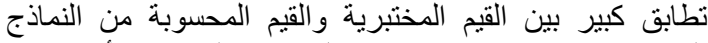

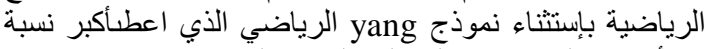

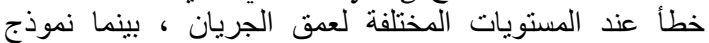
Ghoshal \$ Kundu

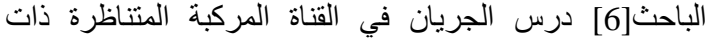

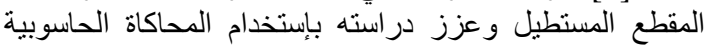

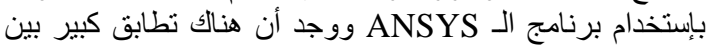

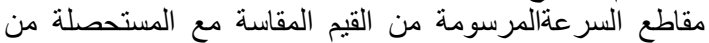

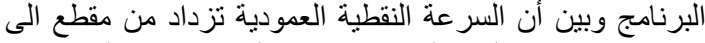

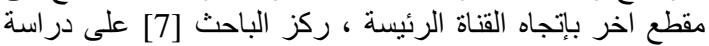

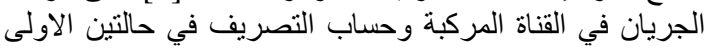

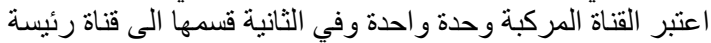

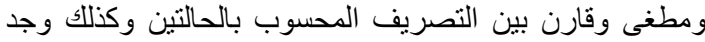

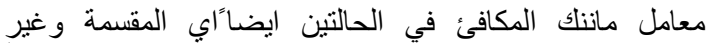

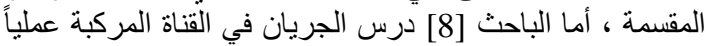

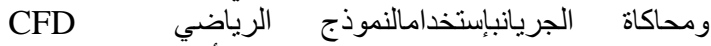
أحد فروع برنامج (Computational Fluid Dynamic) ANSYS

Earsm K- و K-

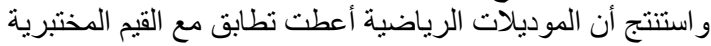

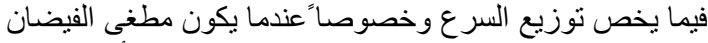

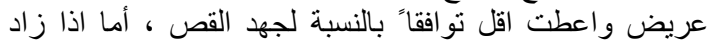

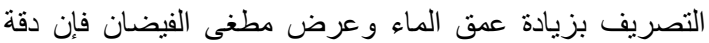

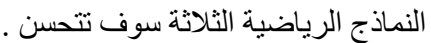

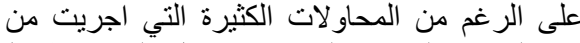

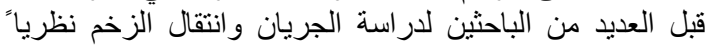

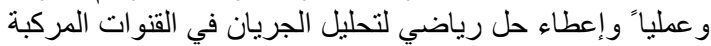

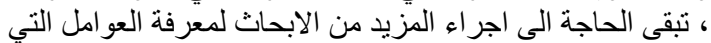

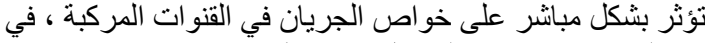
هذا البحث درست هذه مبأن العو امل مختبرياً . اجزاء التجارب المختبرية

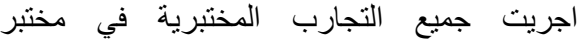
الهيدروليك التابع لقسم السدود و الموارد المائية في كلية الهندسة فئية

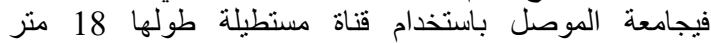

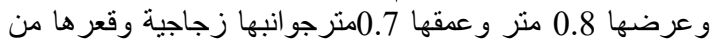

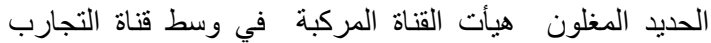

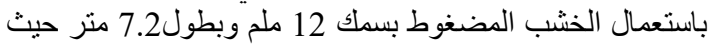

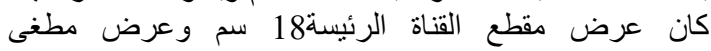

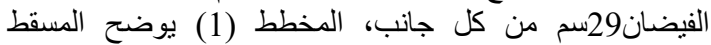

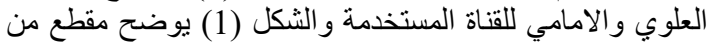

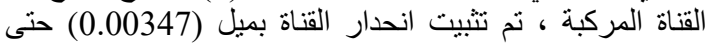

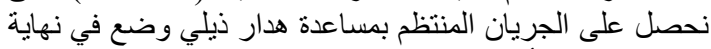

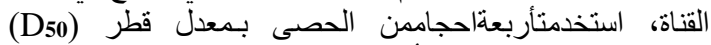

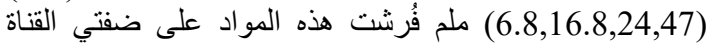

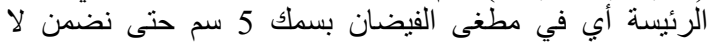

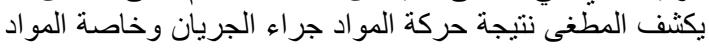

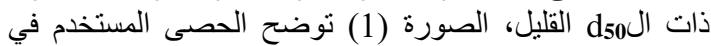
التجارب ،تم قياس السرعة النقطية بعد تقسيم القناة الى ثلى ثلاث

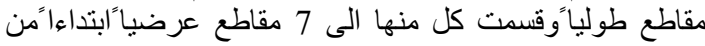

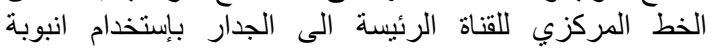
بيتوتالاستاتيكية (34,27.8,19.35,12,7.87) لتراثا كذلك تم فياس منسوب سطح الماء ايضاً بإستخدام المقياس النقطي (19) (point gage)

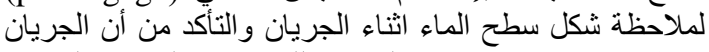

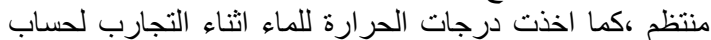

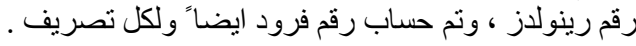




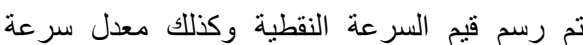

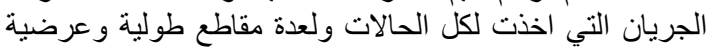

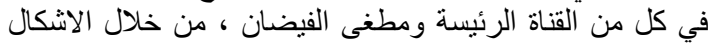
زاد $(3,4)$

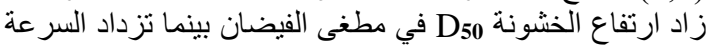

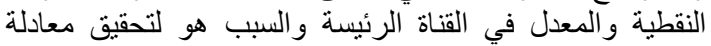

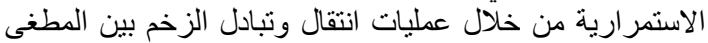
و القناة الرئيسة.

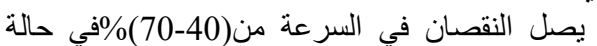

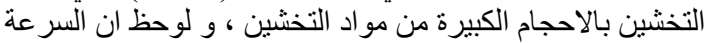

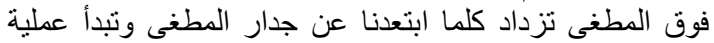

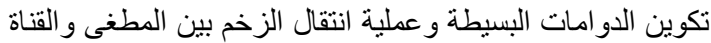

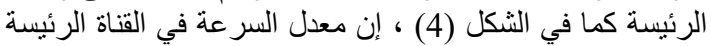

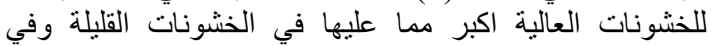

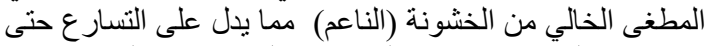

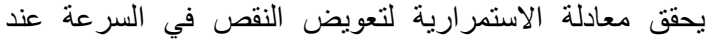

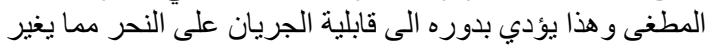

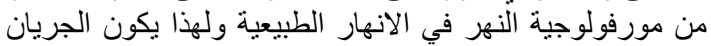

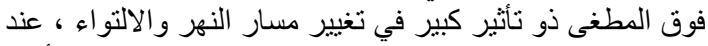

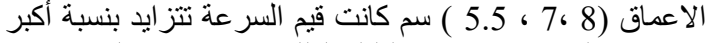

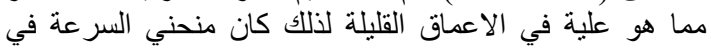

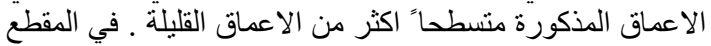

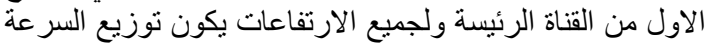

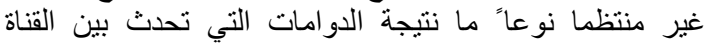

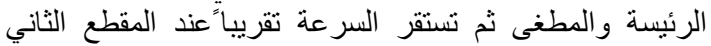

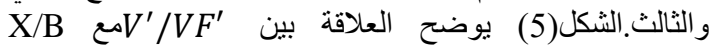

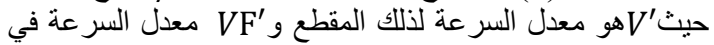

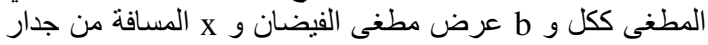

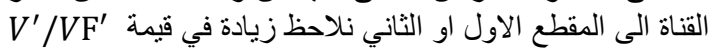

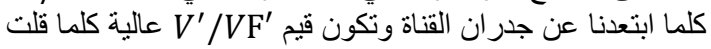

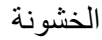
الثكل (6) يوضح العلاقة بين معامل مانتك ورقم

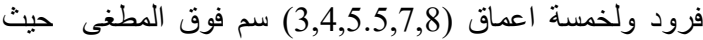

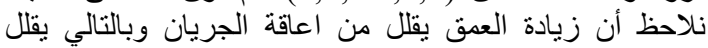
معامل مانتلك و العكس صحيح. يبين الشكل (7) العلاقة بين قيمة معامل ماننك المقاسة

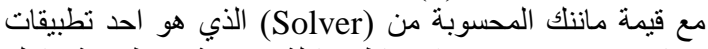
برنامج (Excel) و تم استنباط معادلة وضعية تربط قيمة ماننك

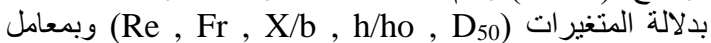
تحديدR مقداره 0.9269 وكانت المعادلة كالتالي :

$$
n=\left(D_{50}\right) \frac{1}{6} * \frac{\left[F r *\left(\frac{x}{b}\right) *\left(\frac{h}{h_{0}}\right)\right]^{0.364}}{\boldsymbol{R e}^{0.349}}+\frac{0.011}{F r^{0.76}} \quad \ldots .(8)
$$

n حيث : D50 Fr :Re

(x/b)

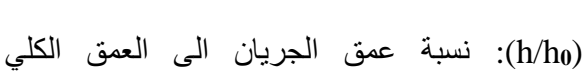

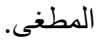
لانجريان.

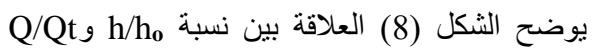
اتضح أن قيمة Q/Qt تزداد بنسبة (25-13)\% للسطح الاملس فيلة

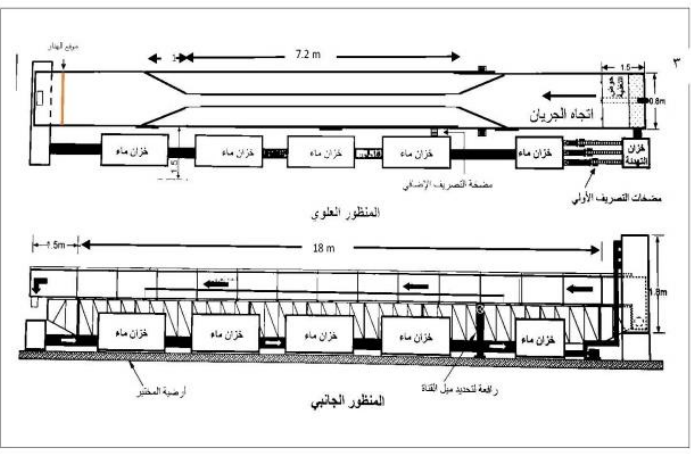

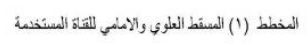
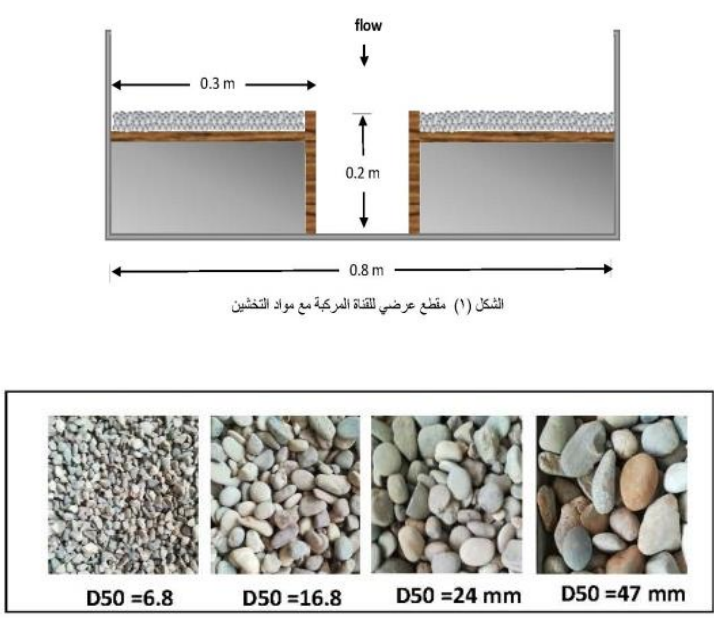

الصورة (1) مواد القعر المستخمة في التخشين أن التر

تم حساب التصريف من معادلة مانتلك:

$Q=\frac{1}{n^{\prime}} A R^{2 / 3} S^{0.5}$

m³/sec التصريف : Q m² مساحة المقطع : : A

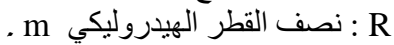

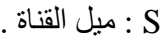

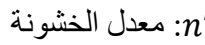

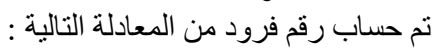

$F r=\frac{V \prime}{\sqrt{g y}}$ حيث:

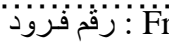

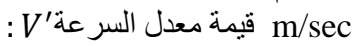

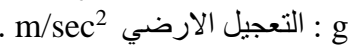
. عمق الجريان عند كل مقطع عموديm : y

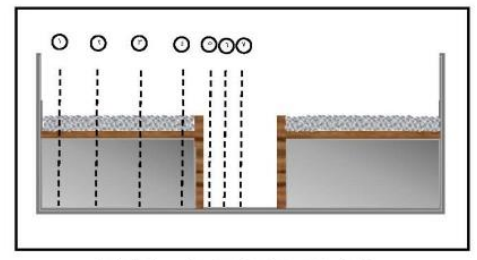

الشكل (؟) مقاطع فياسات السر عثة النقطية 
2- Charles \&Darrien" Study of Flow ina NonSymmetricalCompoundChannel with Rough FloodPlain" Journal - The Institution of Engineers, Malaysia (Vol. 69, No.2, June 2008).

3- Jason W. Kean retal, "Test of a Method to Calculate Near-Bank Velocity and Boundary Shear Stress" Journal of Hydraulic Engineering ASCE / JULY (2009).

4- K.Shiono.etal "The effect of floodplain roughness on flow structures, bedforms and sediment transport rates in meandering channels with over bank flows :part I" j.H.Reasearch (2013) .

5- NeginBinesh

and

HosseinBonakdari"Longitudinal Velocity Distribution in Compound Open Channels: Comparison of Different Mathematical Models" Vol, 8 (9): 1149-1157 (2014) .

6- Rahul Sahoo, "Study of velocity profile of a converging compound channel using Ansys" BSc. thesis pp. 8-27 (2014).

7- Abbas parsaie et al.,"Flow discharge estimation in compound open channel using theoretical approches" Springer (2016).

8- BahramRezaei and AlirezaSafarzade "Numerical modeling of flow field in prismatic compound channels with different floodplain widths " Journal of Applied Research in Water and Wastewater, 3 (2), 2016, 260-270.

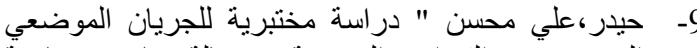

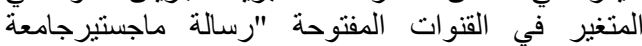

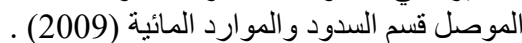

مقارنة مع السطح الخشن وتم ايجاد علاقة بين نسبة التصريف

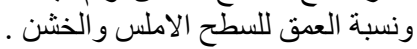

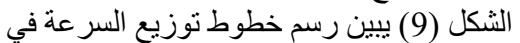

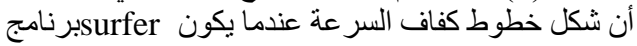
عمق الجريان 8 سم فوق مطنى الفيضان في حالة المطنى غير الأنير

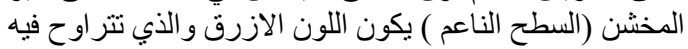

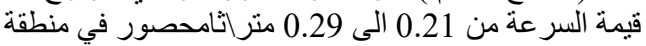

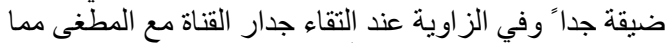

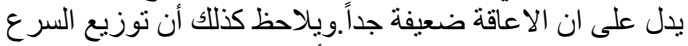

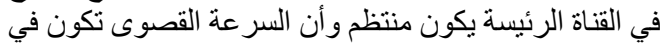

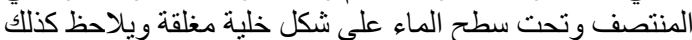

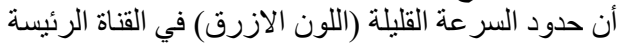

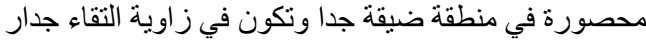

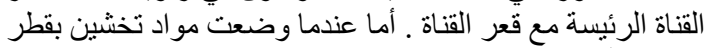

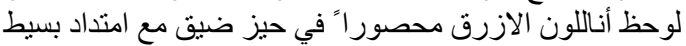

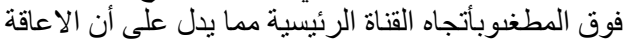

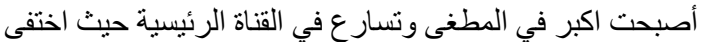

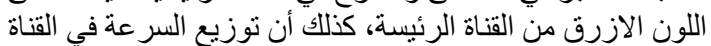

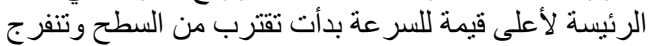

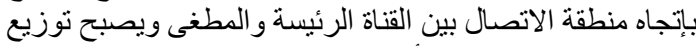

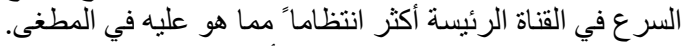

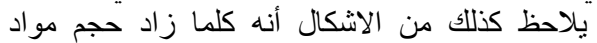

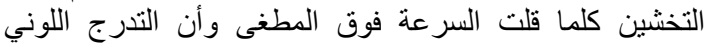

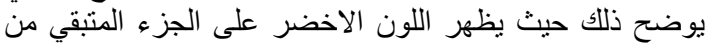

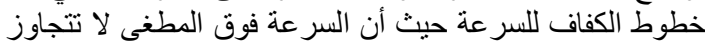

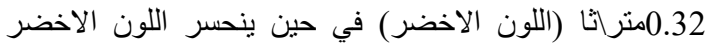

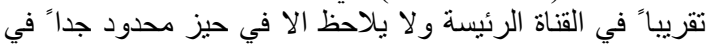

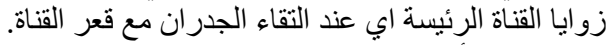
الأستنتاجات الرئرة

تزداد قيمة السرعة مع زيادة العمقات العق ولكن تقل السرعة

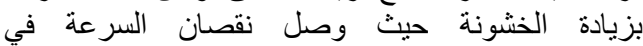

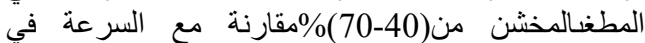
المطغى الناعم.

قيم السرعة تزداد في القناة الرئيسة كلما زادت خشئة المطغي لتحقيق معادلة الاستمر ارية.

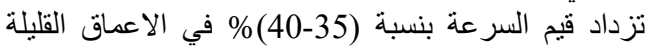

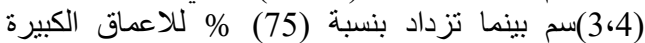
(8،7)سم ولنفس الخشونة.

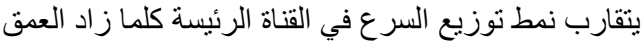

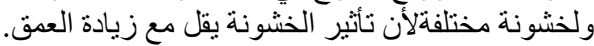

قل مقدار التصريف فوق مطغى الفيضان بمقدار ينر اونة بين (13-25)\% ملاسطحالمخشنة مقارنة بالسطح الناعم.

تم استنباط معادلة وضعية تربط قيمة ماننك بداعلة بلألة

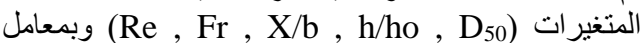
تحديد مقداره 0.9269 ـ 0.

كما لوحظ أن قيمة معامل ماننك نتناسب عكسياً مع رقم

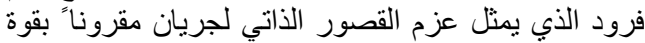

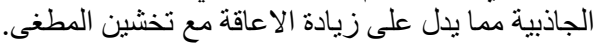

Mahmoud F. Maghrebi and المصادر MajidRahimpour"Streamwisevelocity distribution in irregular shaped channels having composite bed roughness " Flow Measurement and Instrumentation 17 (2006) 237-245. 


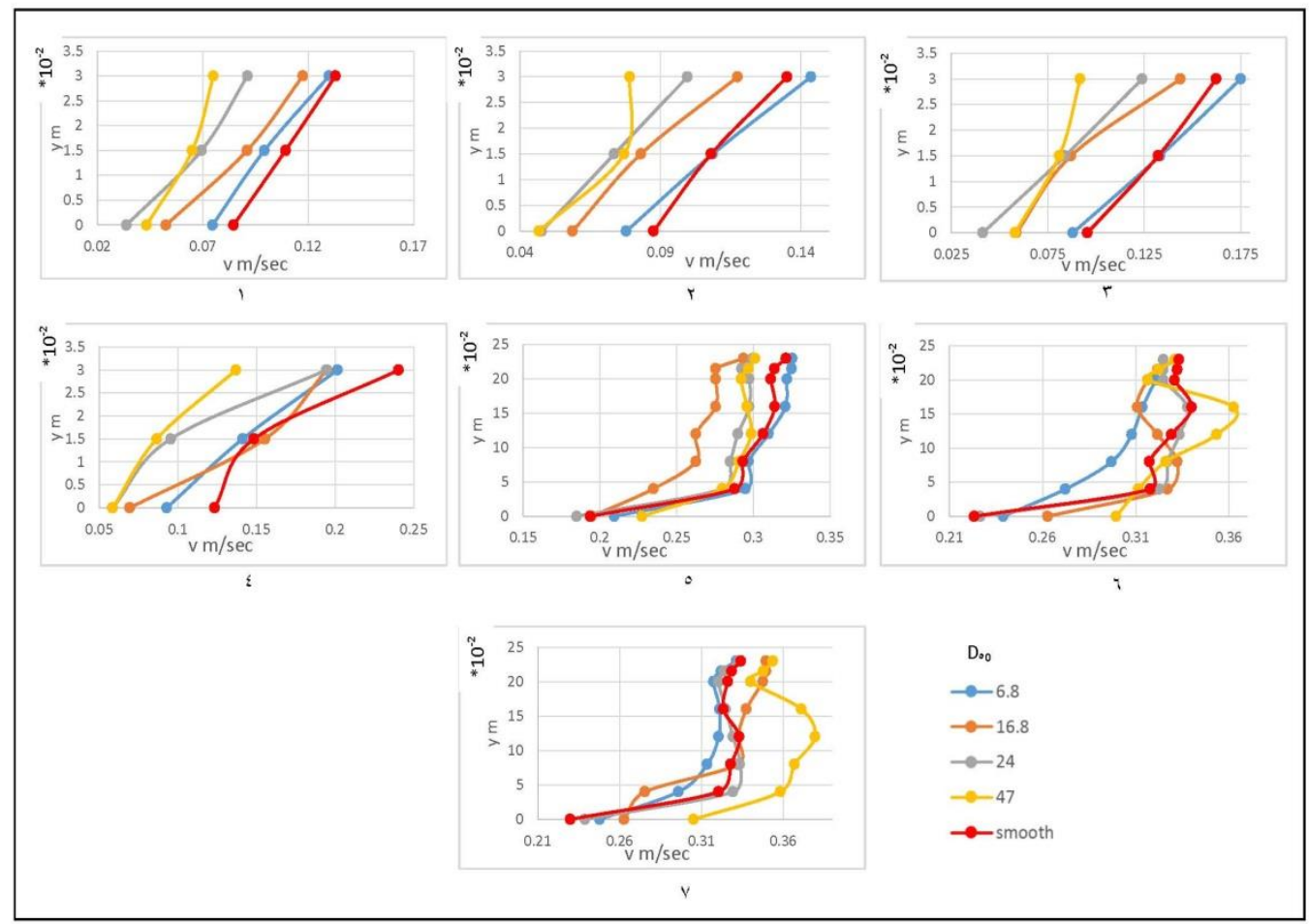

الشكل (3) العلهة بين السرعة مع العقى لسبعة مقاطع لسق ؛ سم وبخشونات مخلفة

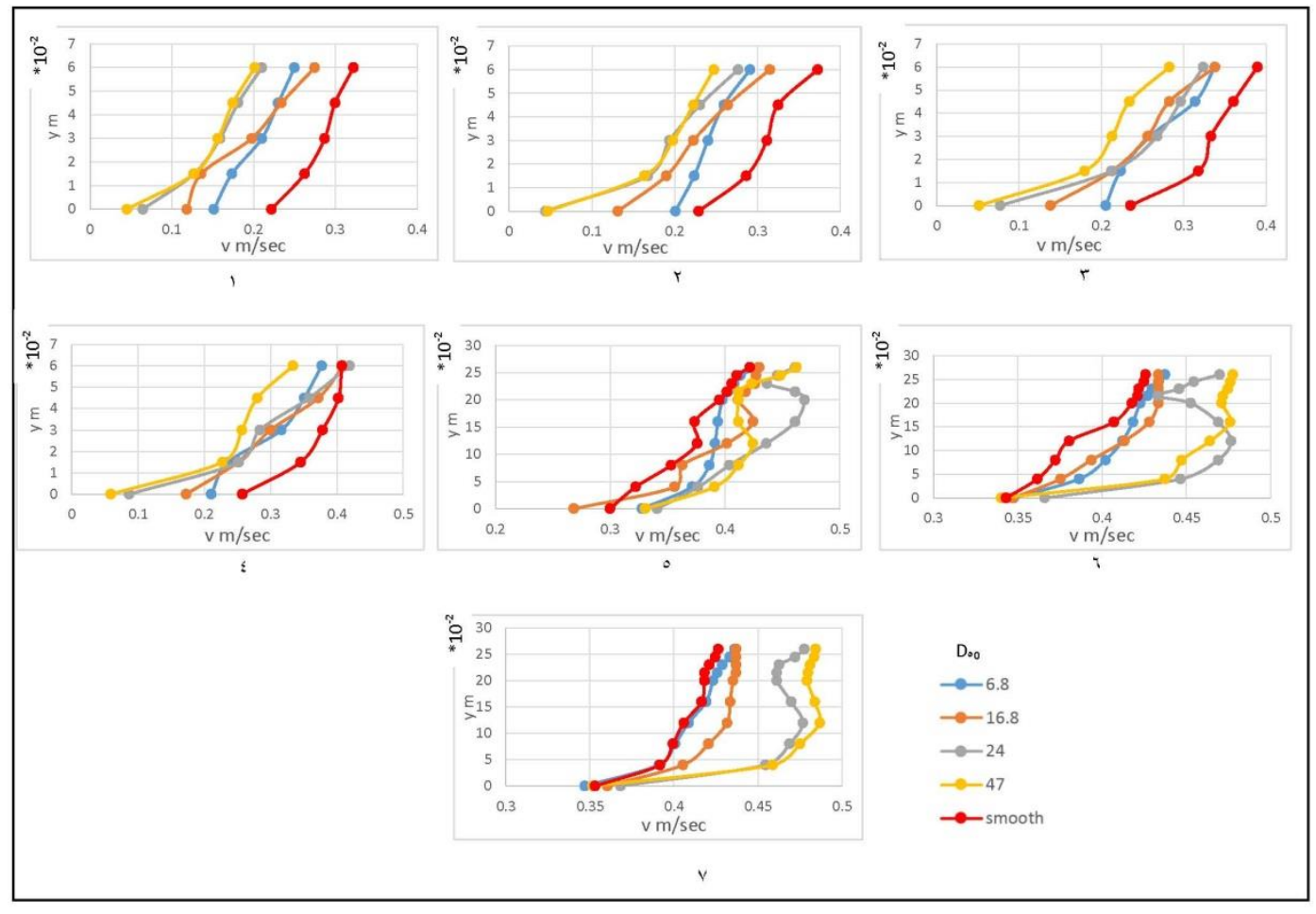

الشكل (4) العلهة بين السرعة مع العق لسبعة مقاطع لعق V سم وبخشونات مختلفة 


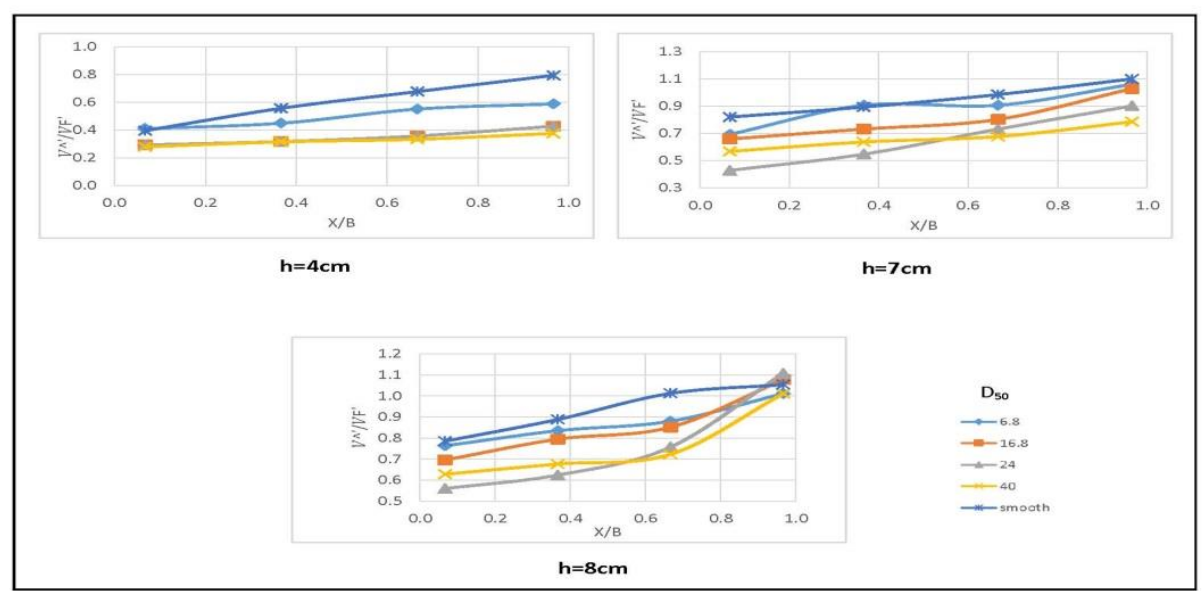

الشكن (0) العلاهة بين

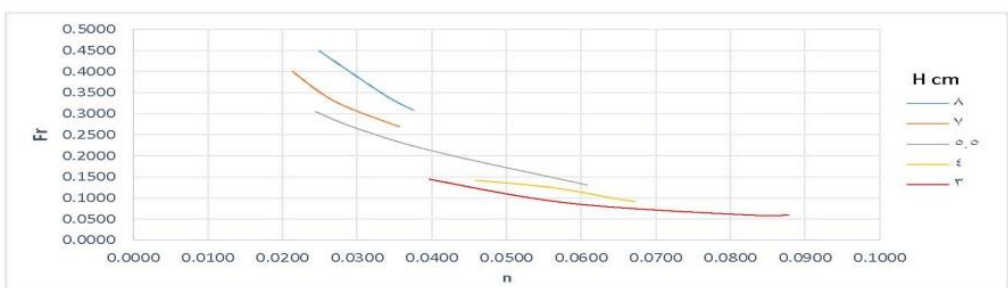

الشكل (飞) العلاهة بين رقم فرود ومعامث مانثلى ولخمس ثصاريف

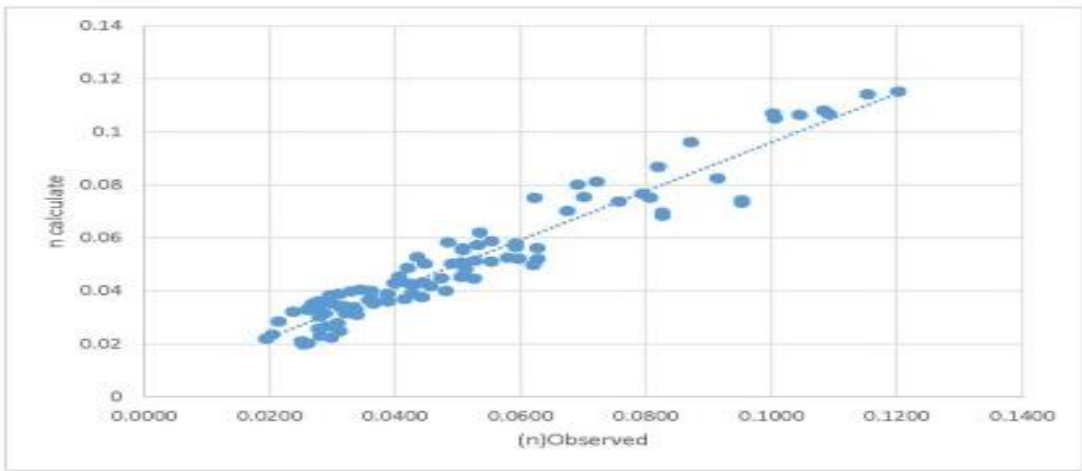

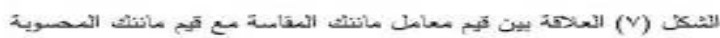

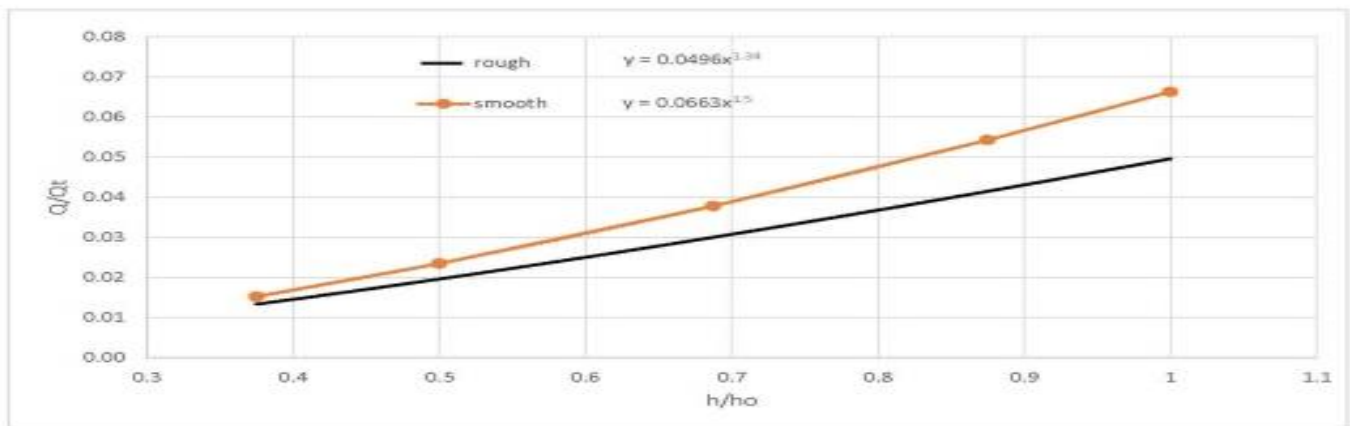

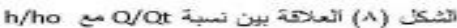




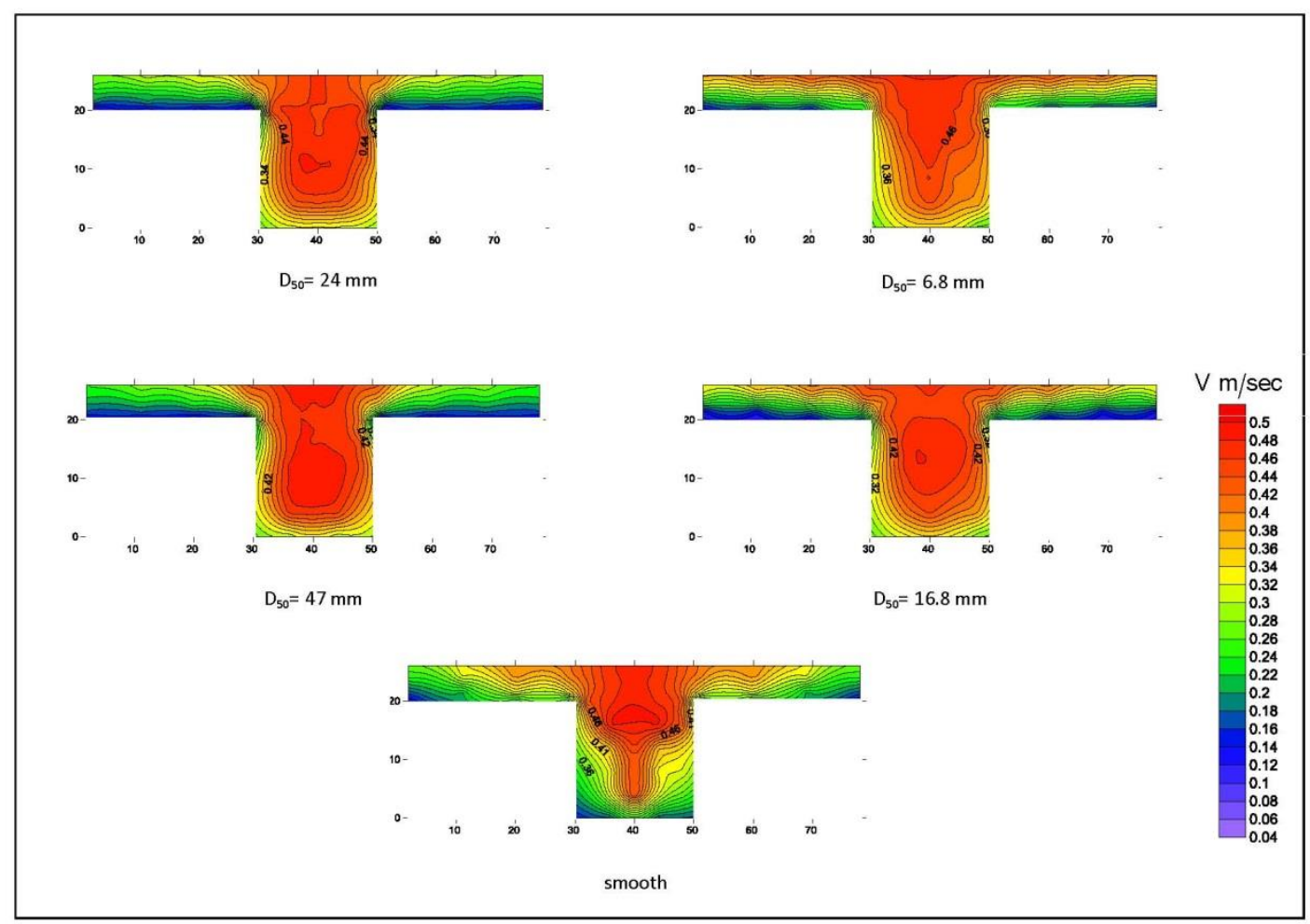

الثشكل (9) خطوط تساوي السرع لعمق ^ سم ولخشونة مختلفة 


\title{
Effect of Roughened Flood Plain of Symmetrical Compound Channel on the Resistance of Flow
}

\author{
AwsYounesHamed \\ M Sc student \\ Dr. MwafaqYounesMhammad \\ Assistant Professor \\ Aws.y@uomosul.edu.iq \\ Mwafaqyounes@gmail.com \\ Department of Dams and water resources Engineering, University of Mosul
}

\begin{abstract}
This research deals with the experimental study of the effect of roughened the flood plain of symmetrical compound channel (having $18 \mathrm{~cm}$ main channel and $29 \mathrm{~cm}$ flood plain width from both sides) on the resistance of flow using different size of gravel roughness $\left(D_{50}=6.8,16.8,24,47\right) \mathrm{mm}$, as well as the flood plain is smooth.

Five discharges $(34,27.8,19.35,12,7.87) \mathrm{L} / \mathrm{sec}$ were carried out for each case, The compound channel was divided to 3 sections perpendicular to the direction of flow and each of them divided to 7 sections , 4 in flood plain and 3 in main channel. Pitot static tube used for velocity measurements and point gage for water surfacelevel measurement as well as the temperature of water was measurement, From the measurements it can be concluded that the velocity over the flood plain was reduced clearly up to $40-70 \%$ when the flood plain roughened by course roughness compare with that of smooth one, while the velocity in the main channel increased to satisfy the continuity equation. Empirical equations were developed to combine the resistance coefficient $n$ with other variables include the flow and geometry characteristics
\end{abstract} Keywords:

compound channel, roughness, velocity distribution, discharge, Froude number 